\title{
FRACTIONAL ORDER PREY-PREDATOR MODEL WITH INFECTED PREDATORS IN THE PRESENCE OF COMPETITION AND TOXICITY
}

\author{
M. R. Lemnaouar ${ }^{1}$, M. Khalfaoui ${ }^{1}$, Y. $_{\text {Louartassi }}^{1,2, *}$ \\ AND I. TOLAIMATE ${ }^{1}$
}

\begin{abstract}
In this paper, we propose a fractional-order prey-predator model with reserved area in the presence of the toxicity and competition. We prove different mathematical results like existence, uniqueness, non negativity and boundedness of the solution for our model. Further, we discuss the local and global stability of these equilibria. Finally, we perform numerical simulations to prove our results.
\end{abstract}

Mathematics Subject Classification. 26A33, 34A08, 34K37.

Received August 4, 2019. Accepted February 7, 2020.

\section{INTRODUCTION}

Each population within an ecosystem does not exist in isolation, and there must be some relationships between these different populations [3]. The relationship between them is divided into types: mutualism, parasitism, competition and predation. The dynamic relationship between predator and prey is long established and will remain among the crucial topics in ecology and mathematical ecology because of its universal existence and its importance [30].

In recent years, the fractional calculations have developed rapidly and have shown broad application prospects in many areas. Useful results can be obtained by extracting a dynamic behaviour of biological systems presented by a mathematical model of integer derivatives. However, most biological systems also have memory. In this case, the modelling in fractional order, unlike the classical mode. The existence of the memory is taken into account. The fractional derivative of a biological process at a point is affected by all the information and behaviour of the model at all previous times, while the classical derivative at a point is only affected by information from the local neighbourhood of that point. For this reason, many papers study the theory of differential fractional equations [19, 21, 23, 24, 26].

Recently, many applications have been developed in areas such as laser physics, chemical reactors, secure communication, biomedicine, epidemiology, signal processing, control theory, mechanics, etc. It has been found that various applications can be modelled using fractional derivatives.

Keywords and phrases: Prey-predator system, toxicity, equilibria, stability, competition, fractional order.

${ }^{1}$ Mohammed V University in Rabat, Superior School of Technology Salé, LASTIMI, Salé, Morocco.

2 Mohammed V University in Rabat, Faculty of Science, Lab-Mia, Rabat, Morocco.

* Corresponding author: ylouartassi@gmail.com 
TABLE 1. Variables and parameters descriptions.

\begin{tabular}{ll}
\hline Parameters and variables & Explanation \\
\hline$x$ & Biomass densities of the unreserved areas \\
$y$ & Biomass densities of the reserved areas \\
$S$ & Susceptible predator \\
$I$ & Infected predator \\
$E_{1}, E_{2}, E_{3}$ & The effort applied for harvesting in the unreserved area, \\
& the susceptible predator populations, the infected predator \\
& populations, respectively \\
$r_{1}, r_{2}$ & The growth rates of fish population inside reserved \\
& and the unserved areas \\
$q_{1}, q_{2}$ & The catchability coefficient in the unreserved area and the \\
& predator species \\
$\sigma_{1}, \sigma_{2}$ & Migration rate from unreserved area to reserved area and \\
$n_{1}, n_{2}$ & reserved area to unreserved area \\
$\gamma$ & The competition coefficients \\
$\delta$ & The strength of intra-specific between prey and infected predator \\
$\beta$ & The disease transmission coefficient \\
$\mu$ & The search rate of the prey toward susceptible predator \\
$\eta$ & The death rate of susceptible predator \\
$\alpha^{\prime}$ & The death rate of infected predator \\
$\sigma$ & Saturation constant while susceptible predators attack the prey \\
$u x^{2}, v y^{2}$ & The conversion rate of susceptible predator due to prey \\
$\frac{\beta x S}{\alpha^{\prime}+x}$ & The reduction terms, in the unreserved area and reserved \\
\hline & area respectively, where $u$ and $v$ the coefficients of toxicity \\
& The functional response of feeding prey by susceptible predator \\
\hline
\end{tabular}

Not long ago, many researchers began to study fractional biological models [1, 10, 20, 25]. In article [14], a dynamic system modelling a prey-predator with harvest area and reserve for prey in the presence of competition and toxicity. In article [25], let us introduce a fractional prey-predator model with two types of susceptible and infected predators. In our paper it has been supposed that the prey are divided into two areas reserved and free and reserved zone, as well as the predators are divided into two categories, susceptible and infected predators. Now the basic model based on $[14,25]$ is governed by the following fractional system (Fig. 1):

$$
\left\{\begin{array}{l}
D^{\alpha} x=r_{1} x\left(1-\frac{x}{K}\right)-\sigma_{1} x+\sigma_{2} y-u x^{2}-\frac{\beta x S}{\alpha^{\prime}+x}-q_{1} E_{1} x-n_{1} x y-\gamma x I \\
D^{\alpha} y=\left(r_{2}-\sigma_{2}\right) y+\sigma_{1} x-v y^{2}-n_{2} x y \\
D^{\alpha} S=\frac{\sigma \beta x S}{\alpha^{\prime}+x}-\delta S I-\mu S-q_{2} E_{2} S \\
D^{\alpha} I=\delta S I+\sigma \gamma x I-q_{3} E_{3} I-\eta I
\end{array}\right.
$$

where $D^{\alpha}$ is in the sense of Caputo fractional derivative and $0<\alpha \leq 1$ defined by [22]: $D^{\alpha} f(t)=$ $\frac{1}{\Gamma(1-\alpha)} \int_{0}^{t} \frac{f^{\prime}(x)}{(t-x)^{\alpha}} \mathrm{d} x$.

Where $f$ is defined by $: f: \Omega \longrightarrow \mathbb{R}^{n}, \Omega \subset \mathbb{R}^{n}$.

The detailed description of the model (1.1) is illustrated in the following schema: the explanation an Units of this parameters and variables given by the tables (Tabs. 1 and 2):

From [7], if there is no migration of fish population from reserved area to unreserved area $\left(\sigma_{2}=0\right)$ and $\left(r_{1}-\sigma_{1}-q_{1} E_{1}<0\right)$, we find that $D^{\alpha} x<0$. Similarly, if there is no migration of fish population from unreserved area to reserved area $\left(\sigma_{1}=0\right)$ and $r_{2}-\sigma_{2}<0$, then $D^{\alpha} y<0$.

If $\sigma \beta-\mu-q_{2} E_{2}<0$, then $D^{\alpha} S<0$. 


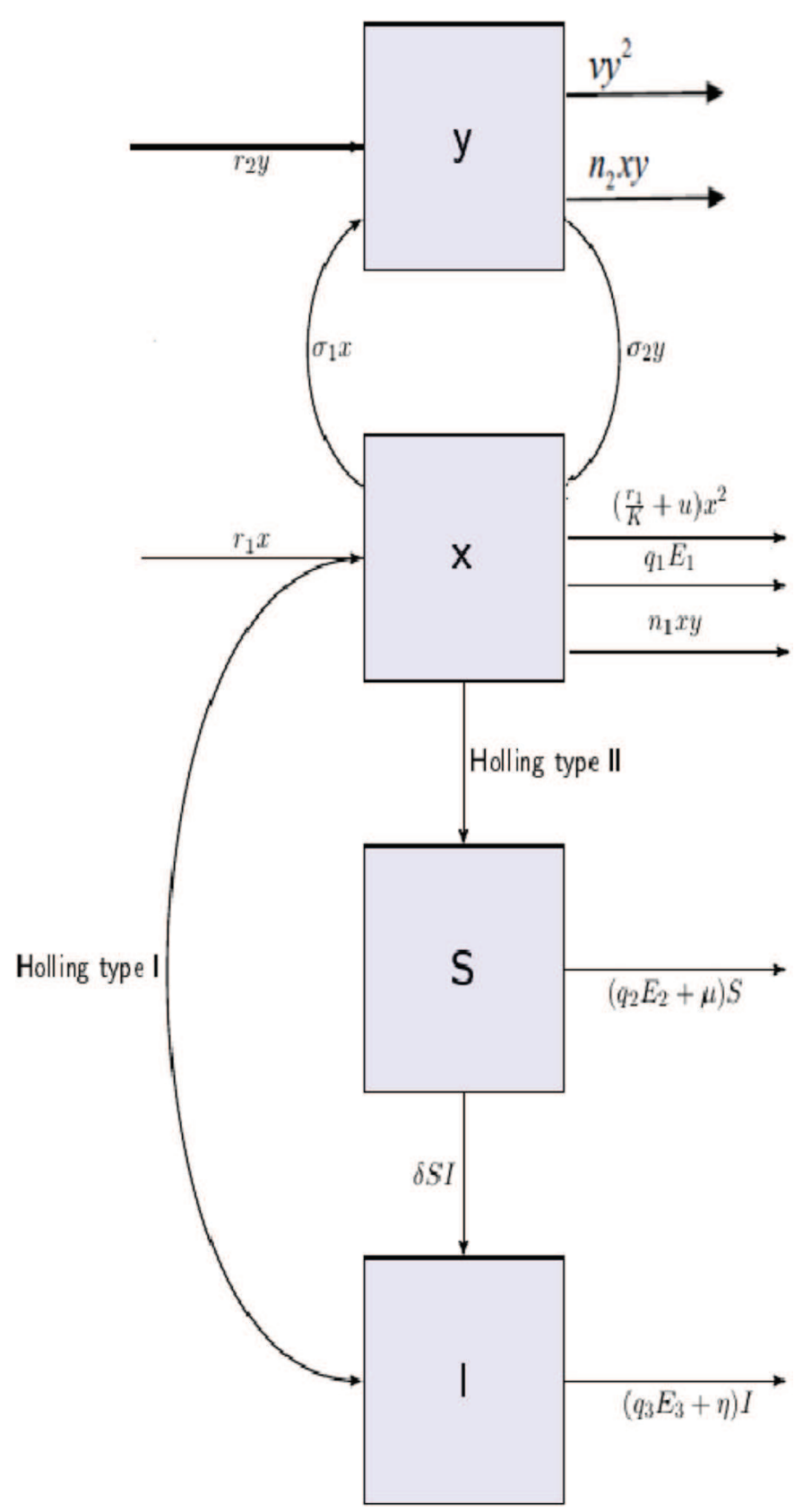

Figure 1. Schematic diagram of the model (1.1).

So, finally we conclude that:

$$
r_{1}-\sigma_{1}-q_{1} E_{1}>0, r_{2}-\sigma_{2}>0, \sigma \beta-\mu-q_{2} E_{2}>0 .
$$

Our paper is organized as follows. In the following section, we prove the of the existence and uniqueness solutions of the system (1.1), in Section 3, we show the boundedness and positivity of the solutions. 
TABLE 2. Units of variables and parameters.

\begin{tabular}{ll}
\hline Parameters and variables & Units \\
\hline$x, y, S, I, \alpha^{\prime}$ & Number per unit area \\
$E_{1}, E_{2}, E_{3}$ & Catch Per Unit Effort \\
$q_{1}, q_{2}$ & Number per unit of fishing effort, \\
$\sigma_{1}, \sigma_{2}, r_{1}, r_{2}, \mu, \eta, \beta, u, v, \delta, n_{1}, n_{2}$ & Per day \\
$\gamma, \sigma$ & Constant \\
\hline
\end{tabular}

In Section 4, we study the existence and stability of all the equilibria of our model (1.1). Finally, we present the numerical simulations to study the stability of the equilibria.

\section{BASIC PROPERTIES AND EQUILIBRIA}

Theorem 2.1. The sufficient condition for the existence and uniqueness of the solution of system (1.1) in the region $\Omega \times\left[t_{0}, T\right]$ with initial conditions

$X(0)=(x(0), y(0), S(0), I(0))$ and $t \in\left[t_{0}, T\right]$ is:

$L=\max \left(\left(r_{1}-q_{1} E_{1}+M\left(n_{1}+\gamma+\beta(1+\sigma)+2\left(\frac{r_{1}}{K}+u\right)\right)\right),\left(r_{2}+M\left(n_{2}+2 v\right)\right)\right.$,

$\left.\left(\beta(1+\sigma)+\mu+q_{2} E_{2}+2 \delta M\right),\left(\sigma \gamma M+\eta+q_{3} E_{3}\right)\right)$.

Proof. Let $X=(x, y, S, I)^{T}$ and $X^{\prime}=\left(x^{\prime}, y^{\prime}, S^{\prime}, I^{\prime}\right)^{T}$ the system (1.1) can be is written in this form:

$$
D^{\alpha} X=F(X),
$$

where

$$
F(X)=\left(\begin{array}{c}
r_{1} x\left(1-\frac{x}{K}\right)-\sigma_{1} x+\sigma_{2} y-u x^{2}-\frac{\beta x S}{\alpha^{\prime}+x}-q_{1} E_{1} x-n_{1} x y-\gamma x I \\
\left(r_{2}-\sigma_{2}\right) y+\sigma_{1} x-v y^{2}-n_{2} x y \\
\frac{\sigma \beta x S}{\alpha^{\prime}+x}-\delta S I-\mu S-q_{2} E_{2} S \\
\delta S I+\sigma \gamma x I-q_{3} E_{3} I-\eta I
\end{array}\right)=\left(\begin{array}{l}
F_{1}(X) \\
F_{2}(X) \\
F_{3}(X) \\
F_{4}(X)
\end{array}\right) \text {. To prove the global }
$$

existence and uniqueness of system (1.1), consider the region $\Omega \times\left[t_{0}, T\right]$,

where $\Omega=\left\{(x, y, S, I) \in \mathbb{R}^{4}: \max \{|x|,|y|,|S|,|I|\} \leq M, M>0\right\}$.

For any $X, X^{\prime} \in \Omega$ :

$$
\begin{aligned}
\left\|F(X)-F\left(X^{\prime}\right)\right\|_{1}= & \sum_{i=1}^{4}\left|F_{i}(X)-F_{i}\left(X^{\prime}\right)\right|, \\
= & \mid\left(r_{1}-\sigma_{1}-q_{1} E_{1}\right)\left(x-x^{\prime}\right)-\left(u+\frac{r_{1}}{K}\right)\left(x^{2}-x^{\prime 2}\right)+\sigma_{2}\left(y-y^{\prime}\right) \\
& -\beta\left(\frac{\alpha^{\prime}\left(x S-x^{\prime} S^{\prime}\right)+x x^{\prime}\left(S-S^{\prime}\right)}{\left(\alpha^{\prime}+x\right)\left(\alpha^{\prime}+x^{\prime}\right)}\right)-n_{1}\left(x y-x^{\prime} y^{\prime}\right)-\gamma\left(x I-x^{\prime} I^{\prime}\right) \mid \\
& +\left|\left(r_{2}-\sigma_{2}\right)\left(y-y^{\prime}\right)+\sigma_{1}\left(x-x^{\prime}\right)-v\left(y^{2}-y^{\prime 2}\right)-n_{2}\left(x y-x^{\prime} y^{\prime}\right)\right| \\
& +\left|\sigma \beta\left(\frac{\alpha^{\prime}\left(x S-x^{\prime} S^{\prime}\right)+x x^{\prime}\left(S-S^{\prime}\right)}{\left(\alpha^{\prime}+x\right)\left(\alpha^{\prime}+x^{\prime}\right)}\right)-\delta\left(S I-S^{\prime} I^{\prime}\right)-\left(\mu+q_{2} E_{2}\right)\left(S-S^{\prime}\right)\right| \\
& +\left|\delta\left(S I-S^{\prime} I^{\prime}\right)+\sigma \gamma\left(x I-x^{\prime} I^{\prime}\right)-\left(\eta+q_{3} E_{3}\right)\left(I-I^{\prime}\right)\right|, \\
\leq & L\left\|X-X^{\prime}\right\|,
\end{aligned}
$$

where $L=\max \left(\left(r_{1}-q_{1} E_{1}+M\left(n_{1}+\gamma+\beta(1+\sigma)+2\left(\frac{r_{1}}{K}+u\right)\right)\right),\left(r_{2}+M\left(n_{2}+2 v\right)\right)\right.$, $\left.\left(\beta(1+\sigma)+\mu+q_{2} E_{2}+2 \delta M\right),\left(\sigma \gamma M+\eta+q_{3} E_{3}\right)\right)$, proving the Lemma.

Thus, $F(X)$ satisfies the Lipschitz's condition [13] with respect to $X$.

Now, we describe the uniform boundedness of the solutions of the system (1.1). 
Lemma 2.2. The set $\Omega^{\prime}=\left\{(x, y, S, I) \in \mathbb{R}_{+}^{4}: x+y+\frac{S}{\sigma}+\frac{I}{\sigma} \leq \frac{H}{\eta+q_{3} E_{3}}\right\}$ is a region of attraction for all solutions initiating in the interior of the positive octant, where

$$
H=\frac{K\left(r_{1}-q_{1} E_{1}+\eta+q_{3} E_{3}\right)^{2}}{4\left(r_{1}+K u\right)}+\frac{\left(r_{2}+\eta+q_{3} E_{3}\right)^{2}}{4 v} .
$$

Proof. We pose $w=x+y+\frac{S}{\sigma}+\frac{I}{\sigma}$,

$$
\begin{aligned}
D^{\alpha} w+\left(\eta+q_{3} E_{3}\right) w= & \left(r_{1}-q_{1} E_{1}+\eta+q_{3} E_{3}\right) x-\left(\frac{r_{1}}{K}+u\right) x^{2}-v y^{2} \\
& +\left(r_{2}+\eta+q_{3} E_{3}\right) y-\left(n_{1}+n_{2}\right) x y+\frac{\left(\eta+q_{3} E_{3}-\mu-q_{2} E_{2}\right) S}{\sigma}
\end{aligned}
$$

Taking $\eta+q_{3} E_{3}<\mu+q_{2} E_{2}$ we get:

$D^{\alpha} w+\left(\eta+q_{3} E_{3}\right) w \leq \frac{K\left(r_{1}-q_{1} E_{1}+\eta+q_{3} E_{3}\right)^{2}}{4\left(r_{1}+K u\right)}+\frac{\left(r_{2}+\eta+q_{3} E_{3}\right)^{2}}{4 v}=H$.

Applying the theory of fractional inequality [22] we get:

$$
w(t) \leq w(0) E_{\alpha}\left(-\left(\eta+q_{3} E_{3}\right) t^{\alpha}\right)+\frac{H}{\eta+q_{3} E_{3}}\left(1-E_{\alpha}\left(-\left(\eta+q_{3} E_{3}\right) t^{\alpha}\right)\right),
$$

where $E_{\alpha}(z)=\sum_{k=0}^{\infty} \frac{z^{k}}{\Gamma(\alpha k+1)}$ is Mittag-Leffler function [22], $\Gamma(z)=\int_{0}^{\infty} x^{z-1} e^{-x} \mathrm{~d} x$ is Euler's Gamma function, and $0<E_{\alpha}\left(-\left(\eta+q_{3} E_{3}\right) t^{\alpha}\right) \leq 1$, if $t \rightarrow \infty$, we have $0<w(t) \leq w(0)+\frac{H}{\eta+q_{3} E_{3}}$, proving this Lemma.

Now, we find the positive equilibria, then we study their local stability. We denote the function on the right hand side of the system (1.1) by $F_{i}(x, y, S, I)$, for $i=1, \ldots, 4$.

Equilibria of model (1.1) is obtained by solving $F_{i}(x, y, S, I)=0$, for $i=1, \ldots, 4$. Then, we find that our model (1.1) admits five positive equilibria:

1. $P_{0}(0,0,0,0)$ there is a trivial equilibrium.

2. $P_{1}\left(x_{1}, y_{1}, 0,0\right)$, where $\left(x_{1}, y_{1}\right)$ is the positive solution of the following equations:

$$
\begin{array}{ll}
\left(r_{1}-\sigma_{1}-q_{1} E_{1}\right) x-\left(\frac{r_{1}+K u}{K}\right) x^{2}+\sigma_{2} y-n_{1} x y & =0, \\
\left(r_{2}-\sigma_{2}\right) y+\sigma_{1} x-v y^{2}-n_{2} x y & =0 .
\end{array}
$$

Using system (2.2), $x$ is satisfied by the following equation,

$$
a_{3} x^{3}+a_{2} x^{2}+a_{1} x+a_{0}=0,
$$

where:

$$
\begin{aligned}
a_{3}= & \left(u+\frac{r_{1}}{K}\right)\left(n_{1} n_{2}-v\left(u+\frac{r_{1}}{K}\right)\right), \\
a_{2}= & \frac{2 v\left(r_{1}+K u\right)\left(r_{1}-\sigma_{1}-q_{1} E_{1}\right)}{K}-n_{2} \sigma_{2}\left(\frac{r_{1}}{K}+u\right)-n_{1} n_{2}\left(r_{1}-\sigma_{1}-q_{1} E_{1}\right) \\
& -n_{1}\left(r_{2}-\sigma_{2}\right)\left(u+\frac{r_{1}}{K}\right)+\sigma_{1} n_{1}^{2}, \\
a_{1}= & -v\left(r_{1}-\sigma_{1}-q_{1} E_{1}\right)^{2}+\left(r_{1}-\sigma_{1}-q_{1} E_{1}\right)\left(n_{2} \sigma_{2}+n_{1}\left(r_{2}-\sigma_{2}\right)\right) \\
& -2 \sigma_{1} \sigma_{2} n_{1}+\left(r_{2}-\sigma_{2}\right) \sigma_{2}\left(u+\frac{r_{1}}{K}\right), \\
a_{0}= & -\sigma_{2}\left(r_{2}-\sigma_{2}\right)\left(r_{1}-\sigma_{1}-q_{1} E_{1}\right)+\sigma_{1} \sigma_{2}^{2} .
\end{aligned}
$$


Using criteria of Descartes [5], the above equation (2.3) had a unique positive solution if the following inequalities hold:

$$
\begin{array}{rll}
a_{0}>0 \quad \text { if } \quad & \left(r_{2}-\sigma_{2}\right)\left(r_{1}-\sigma_{1}-q_{1} E_{1}\right)<\sigma_{1} \sigma_{2}, \\
a_{1}>0 \text { if } \quad & \left(r_{1}-\sigma_{1}-q_{1} E_{1}\right)\left(n_{2} \sigma_{2}+n_{1}\left(r_{2}-\sigma_{2}\right)\right) \\
& +\left(r_{2}-\sigma_{2}\right) \sigma_{2}\left(u+\frac{r_{1}}{K}\right)>2 \sigma_{1} \sigma_{2} n_{1}+v\left(r_{1}-\sigma_{1}-q_{1} E_{1}\right)^{2} .
\end{array}
$$

Then,

$$
E_{1}>\frac{1}{q_{1}} \max \left(r_{1}-\sigma_{1}-\frac{\sigma_{1} \sigma_{2}}{r_{2}-\sigma_{2}}, r_{1}-\sigma_{1}-\frac{\sqrt{\Delta_{1}+n_{2} \sigma_{2}+n_{1}\left(r_{2}-\sigma_{2}\right)}}{2 v}\right),
$$

where $\Delta_{1}=\left(n_{2} \sigma_{2}+n_{1}\left(r_{2}-\sigma_{2}\right)\right)^{2}+4 v\left(u+\frac{r_{1}}{K}\right)\left(r_{2}-\sigma_{2}\right) \sigma_{2}$

$$
\begin{array}{llll}
a_{2}>0 & \text { if } & v\left(r_{1}-\sigma_{1}-q_{1} E_{1}\right)>n_{2} \sigma_{2}+n_{1}\left(r_{2}-\sigma_{2}\right), \\
a_{3}<0 & \text { if } & n_{1} n_{2}<v\left(u+\frac{r_{1}}{K}\right) .
\end{array}
$$

Then

$$
y_{1}=\frac{x_{1}}{\sigma_{2}-n_{1} x_{1}}\left(\left(\frac{r_{1}+K u}{K}\right) x_{1}-\left(r_{1}-\sigma_{1}-q_{1} E_{1}\right)\right)>0
$$

if

$$
\frac{\left(r_{1}-\sigma_{1}-q_{1} E_{1}\right) K}{r_{1}+K u}<x_{1}<\frac{\sigma_{2}}{n_{1}} \quad \text { or } \quad \frac{\sigma_{2}}{n_{1}}<x_{1}<\frac{\left(r_{1}-\sigma_{1}-q_{1} E_{1}\right) K}{r_{1}+K u} .
$$

3. In the interior of the equilibrium $P_{2}\left(x_{2}, y_{2}, 0, I_{2}\right)$, i.e. $F_{i}\left(x_{2}, y_{2}, 0, I_{2}\right)=0, i=1,2,4$, we get a positive solution:

$$
\begin{aligned}
x_{2} & =\frac{\eta+q_{3} E_{3}}{\sigma \gamma} \\
y_{2} & =\frac{r_{2}-\sigma_{2}-n_{2} x_{2}+\sqrt{\left(r_{2}-\sigma_{2}-n_{2} x_{2}\right)^{2}+4 \sigma_{1} x_{2} v}}{2 v}, \\
I_{2} & =\frac{1}{\gamma x_{2}}\left(\left(r_{1}-\sigma_{1}-n_{1} y_{2}-q_{1} E_{1}\right) x_{2}-\left(\frac{r_{1}}{K}+u\right) x_{2}^{2}+\sigma_{2} y_{2}\right)>0 .
\end{aligned}
$$

if

$$
0<x_{2}<\frac{r_{1}-\sigma_{1}-q_{1} E_{1}-n_{1} y_{2}+\sqrt{\left(r_{1}-\sigma_{1}-n_{1} y_{2}-q_{1} E_{1}\right)^{2}+4 \sigma_{2}\left(u+\frac{r_{1}}{K}\right) y_{2}}}{2\left(\frac{r_{1}}{K}+u\right)} .
$$


4. In the interior of the equilibrium $P_{3}\left(x_{3}, y_{3}, S_{3}, 0\right)$, i.e. $F_{i}\left(x_{3}, y_{3}, S_{3}, 0\right)=0, i=1,2,3$, we get a positive solution:

$$
\begin{aligned}
x_{3} & =\frac{\alpha^{\prime}\left(\mu+q_{2} E_{2}\right)}{\sigma \beta-\left(\mu+q_{2} E_{2}\right)}>0, \quad \text { if } \sigma \beta>\mu+q_{2} E_{2}, \\
y_{3} & =\frac{\left(r_{2}-\sigma_{2}-n_{2} x_{3}\right)+\sqrt{\left(r_{2}-\sigma_{2}-n_{2} x_{3}\right)^{2}+4 v \sigma_{1} x_{3}}}{2 v}, \\
S_{3} & =\frac{\alpha^{\prime}+x_{3}}{\beta x_{3}}\left(\left(r_{1}-\sigma_{1}-q_{1} E_{1}-n_{1} y_{3}\right) x_{3}+\sigma_{2} y_{3}-\left(u+\frac{r_{1}}{K}\right) x_{3}^{2}\right) .
\end{aligned}
$$

$S_{3}>0 \quad$ if $\quad 0<x_{3}<\frac{r_{1}-\sigma_{1}-q_{1} E_{1}-n_{1} y_{3}+\sqrt{\left(r_{1}-\sigma_{1}-n_{1} y_{3}-q_{1} E_{1}\right)^{2}+4 \sigma_{2}\left(u+\frac{r_{1}}{K}\right) y_{3}}}{2\left(\frac{r_{1}}{K}+u\right)}$.

5. Using $F_{i}\left(x_{4}, y_{4}, S_{4}, I_{4}\right)=0, i=1, \ldots, 4$, the equilibrium point $P_{4}\left(x_{4}, y_{4}, S_{4}, I_{4}\right)$ is given by:

$$
\begin{aligned}
& y_{4}=\frac{r_{2}-\sigma_{2}-n_{2} x_{4}+\sqrt{\left(r_{2}-\sigma_{2}-n_{2} x_{4}\right)^{2}+4 \sigma_{1} x_{4} v}}{2 v} \\
& S_{4}=\frac{\eta+q_{3} E_{3}-\sigma \gamma x_{4}}{\delta}>0, \quad \text { if } \quad x_{4}<\frac{\eta+q_{3} E_{3}}{\sigma \gamma}, \\
& I_{4}=\frac{1}{\delta}\left(\frac{\sigma \beta x_{4}}{\alpha^{\prime}+x_{4}}-\left(\mu+q_{2} E_{2}\right)\right)>0, \quad \text { if } \quad x_{4}>\frac{\alpha^{\prime}\left(\mu+q_{2} E_{2}\right)}{\sigma \beta-\alpha^{\prime}\left(\mu+q_{2} E_{2}\right)} .
\end{aligned}
$$

where $\left(x_{4}, y_{4}\right)$ is the positive solution of the following equations:

$$
\begin{array}{ll}
r_{1} x\left(1-\frac{x}{K}\right)-\sigma_{1} x+\sigma_{2} y-u x^{2}-\frac{\beta x S}{\alpha^{\prime}+x}-q_{1} E_{1} x-n_{1} x y-\gamma x I & =0, \\
\left(r_{2}-\sigma_{2}\right) y+\sigma_{1} x-v y^{2}-n_{2} x y & =0 .
\end{array}
$$

After the calculations, $x$ is satisfied by the following equation,

$$
b_{5} x^{5}+b_{4} x^{4}+b_{3} x^{3}+b_{2} x^{2}+b_{1} x+b_{0}=0,
$$

where

$$
\begin{aligned}
b_{5}= & b n_{2} n_{1}-b^{2} v \\
b_{4}= & 2 a b v-a n_{2} n_{1}-2 b^{2} \alpha^{\prime} v-b d n_{1}+2 b \alpha^{\prime} n_{2} n_{1}-b \sigma_{2} n_{2}+\sigma_{1} n_{1}^{2}, \\
b_{3}= & -a^{2} v+4 a b \alpha^{\prime} v+a d n_{1}-2 a \alpha^{\prime} n_{2} n_{1}+a \sigma_{2} n_{2}-b^{2} \alpha^{\prime 2} v-2 b c \beta v-2 b d \alpha^{\prime} v \\
& +b d \sigma_{2}+b \alpha^{\prime 2} n_{2} n_{1}-2 b \alpha^{\prime} \sigma_{2} n_{2}+c \beta n_{2} n_{1}+2 \alpha^{\prime} \sigma_{1} n_{1}^{2}-2 \sigma_{1} \sigma_{2} n_{1}, \\
b_{2}= & -2 a^{2} \alpha^{\prime} v+2 a b \alpha^{\prime 2} v+2 a c \beta v+2 a d \alpha^{\prime} n_{1}-a d \sigma_{2}-a \alpha^{\prime 2} n_{2} n_{1}+2 a \alpha^{\prime} \sigma_{2} n_{2} \\
& -2 b c \alpha^{\prime} \beta v-b d \alpha^{\prime 2} n_{1}+2 b d \alpha^{\prime} \sigma_{2}-b \alpha^{\prime 2} \sigma_{2} n_{2}-c d \beta n_{1}+c \alpha^{\prime} v n_{2} n_{1}-c \beta \sigma_{2} n_{2} \\
& +\alpha^{\prime 2} \sigma_{1} n_{1}^{2}-4 \alpha^{\prime} \sigma_{1} \sigma_{2} n_{1}+\sigma_{1} \sigma_{2}^{2}, \\
b_{1}= & -a^{2} \alpha^{\prime 2} v+2 a c \alpha^{\prime} \beta v+a d \alpha^{\prime 2} n_{1}-2 a d \alpha^{\prime} \sigma_{2}+a \alpha^{\prime 2} \sigma_{2} n_{2}+b d \alpha^{\prime 2} \sigma_{2}-c^{2} \beta^{2} v \\
& -c d \alpha^{\prime} \beta n_{1}+c d \beta \sigma_{2}-c \alpha^{\prime} \beta \sigma_{2} n_{2}-2 \alpha^{\prime 2} \sigma_{1} \sigma_{2} n_{1}+2 \alpha^{\prime} \sigma_{1} \sigma_{2}^{2}, \\
b_{0}= & -a d \alpha^{\prime 2} \sigma_{2}+c d \alpha^{\prime} \beta \sigma_{2}+\alpha^{\prime 2} \sigma_{2}^{2}, \\
a= & r_{1}-\sigma_{1}-q_{1} E_{1}+\frac{\gamma}{\delta}\left(\mu+q_{2} E_{2}\right), \\
b= & \frac{r_{1}}{K}+u \\
c= & \frac{\eta+q_{3} E_{3}}{\delta} \\
d= & r_{2}-\sigma_{2} .
\end{aligned}
$$

Using the criteria of Descartes [5] it is necessary to impose that: 


$$
\frac{\alpha^{\prime}\left(\mu+q_{2} E_{2}\right)}{\sigma \beta-\alpha^{\prime}\left(\mu+q_{2} E_{2}\right)}<x_{4}<\frac{\eta+q_{3} E_{3}}{\sigma \gamma}, b_{5}<0 \text { and } b_{i}>0 \text {, for } i=0, \ldots, 4 \text {. }
$$

To study the local stability of equilibria, the eigenvalues of the Jacobian matrix of system (1.1) are computed by: $J(x, y, S, I)=\left(\begin{array}{cccc}J_{11} & J_{12} & J_{13} & J_{14} \\ J_{21} & J_{22} & 0 & 0 \\ J_{31} & 0 & J_{33} & J_{34} \\ J_{41} & 0 & J_{43} & J_{44}\end{array}\right)$, where:

$$
\begin{aligned}
& J_{11}=r_{1}-\sigma_{1}-q_{1} E_{1}-2\left(\frac{r_{1}}{K}+u\right) x-n_{1} y-\gamma I-\frac{\beta S \alpha^{\prime}}{\left(\alpha^{\prime}+x\right)^{2}}, \\
& J_{12}=\sigma_{2}-n_{1} x, \\
& J_{13}=\frac{-\beta x}{\alpha^{\prime}+x} \\
& J_{14}=-\gamma x \\
& J_{21}=\sigma_{1}-n_{2} y \\
& J_{22}=r_{2}-\sigma_{2}-2 v y-n_{2} x, \\
& J_{31}=\frac{\sigma \beta \alpha^{\prime} S}{\left(\alpha^{\prime}+x\right)^{2}} \\
& J_{33}=\frac{\sigma \beta x}{\alpha^{\prime}+x}-\delta I-\mu-q_{2} E_{2}, \\
& J_{34}=-\delta S \\
& J_{41}=\sigma \gamma I \\
& J_{43}=\delta I \\
& J_{44}=\delta S+\sigma \gamma x-q_{3} E_{3}-\eta .
\end{aligned}
$$

Theorem 2.3. The equilibrium $P_{0}(0,0,0,0)$ of the system (1.1) is unstable.

Proof. The characteristic equation of $P_{0}(0,0,0,0)$ is

$$
\begin{aligned}
& {\left[\lambda^{2}-\left(r_{1}-\sigma_{1}+r_{2}-\sigma_{2}-q_{1} E_{1}\right) \lambda+\left(r_{2}-\sigma_{2}\right)\left(r_{1}-\sigma_{1}-q_{1} E_{1}\right)-\sigma_{2} \sigma_{1}\right]} \\
& \times\left(\lambda+\mu+q_{2} E_{2}\right)\left(\lambda+\eta+q_{3} E_{3}\right)=0 .
\end{aligned}
$$

Then, the eigenvalues of matrix (2.15) to the equilibrium point $P_{0}$ :

$\lambda_{1}=-\left(\eta+q_{3} E_{3}\right)<0, \lambda_{2}=-\left(\mu+q_{2} E_{2}\right)<0$, and $\lambda_{3}+\lambda_{4}=r_{1}-\sigma_{1}-q_{1} E_{1}+r_{2}-\sigma_{2}>0$. Therefore one of the eigenvalues $\lambda_{3}$ and $\lambda_{4}$ not satisfy Matignon's condition [17]. Hence, $P_{0}(0,0,0,0)$ is unstable.

Theorem 2.4. The equilibrium point $P_{1}\left(x_{1}, y_{1}, 0,0\right)$ of the system (1.1) is locally asymptotically stable if $x_{1}<\min \left(\frac{\eta+q_{3} E_{3}}{\sigma \gamma}, \frac{\alpha^{\prime}\left(\mu+q_{2} E_{2}\right)}{\sigma \beta-\left(\mu+q_{2} E_{2}\right)}\right)$ and (2.16) satisfied.

(1) If $\Delta>0, P_{1}$ is locally asymptically stable.

(2) If $\Delta<0$, and $\left|\arg \left(\lambda_{3,4}\right)\right|>\frac{\alpha \pi}{2}, \quad P_{1}$ is locally asymptically stable.

Proof. From (2.15) evaluated at equilibrium point $P_{1}$, the characteristic equation is:

$\left(\lambda^{2}+s \lambda+p\right)\left(\lambda-\sigma \gamma x_{1}+\eta+q_{3} E_{3}\right)\left(\lambda-\frac{\sigma \beta x_{1}}{\alpha^{\prime}+x_{1}}+\mu+q_{2} E_{2}\right)=0$.

Where

$$
\begin{aligned}
& s=\left(\sigma_{2} \frac{y_{1}}{x_{1}}+\left(u+\frac{r_{1}}{K}\right) x_{1}+\sigma_{1} \frac{x_{1}}{y_{1}}+v y_{1}\right), \\
& p=\left(\sigma_{2} \frac{y_{1}}{x_{1}}+\left(u+\frac{r_{1}}{K}\right) x_{1}\right)\left(\sigma_{1} \frac{x_{1}}{y_{1}}+v y_{1}\right)-\left(\sigma_{2}-n_{1} x_{1}\right)\left(\sigma_{1}-n_{2} y_{1}\right) .
\end{aligned}
$$

Therefore, the first and second eigenvalues are:

$$
\begin{aligned}
& \lambda_{1}=\sigma \gamma x_{1}-\left(\eta+q_{3} E_{3}\right)<0, \text { if } x_{1}<\frac{\eta+q_{3} E_{3}}{\sigma \gamma}, \text { then }\left|\arg \left(\lambda_{1}\right)\right|=\pi>\frac{\alpha \pi}{2} . \\
& \lambda_{2}=\frac{\sigma \beta x_{1}}{\alpha^{\prime}+x_{1}}-\left(\mu+q_{2} E_{2}\right)<0, \text { if } x_{1}<\frac{\alpha^{\prime}\left(\mu+q_{2} E_{2}\right)}{\sigma \beta-\left(\mu+q_{2} E_{2}\right)}, \text { then }\left|\arg \left(\lambda_{1}\right)\right|=\pi>\frac{\alpha \pi}{2} .
\end{aligned}
$$


We pose $\Delta=s^{2}-4 p$. If $\Delta>0, \lambda_{3}$ and $\lambda_{4}$ are purely real and negative.

If $\Delta<0, \lambda_{3}$ and $\lambda_{4}$ are complex number and if $\left|\arg \left(\lambda_{3,4}\right)\right|=\tan ^{-1}\left(\frac{\sqrt{-\Delta}}{s}\right)>\frac{\alpha \pi}{2}$. Hence, $P_{1}$ is locally asymptotically stable.

Theorem 2.5. The equilibrium point $P_{2}\left(x_{2}, y_{2}, 0, I_{2}\right)$ is locally asymptotically stable if $x_{2}<\frac{\alpha^{\prime}\left(\delta I_{2}+\mu+q_{2} E_{2}\right)}{\sigma \beta-\alpha^{\prime}\left(\delta I_{2}+\mu+q_{2} E_{2}\right)}$ and (2.17) satisfied.

Proof. From the Jacobian matrix $J\left(x_{2}, y_{2}, 0, I_{2}\right)$, the characteristic equation at $P_{2}$ :

$\left(\lambda-\left(\frac{\sigma \beta x_{2}}{\alpha^{\prime}+x_{2}}-\left(\delta I_{2}+\mu+q_{2} E_{2}\right)\right)\right)\left(\lambda^{3}+c_{2} \lambda^{2}+c_{1} \lambda+c_{0}\right)=0$, where:

$c_{2}=-\left(A_{1}+B_{1}\right)$,

$c_{1}=A_{1} B_{1}-\left(\sigma_{1}-n_{2} y_{2}\right)\left(\sigma_{2}-n_{1} x_{2}\right)+\sigma \gamma^{2} I_{2} x_{2}$,

$c_{0}=-B_{1} \sigma \gamma^{2} I_{2} x_{2}$,

$A_{1}=r_{1}-\sigma_{1}-q_{1} E_{1}-2\left(\frac{r_{1}+K u}{K}\right) x_{2}-n_{1} y_{2}-\gamma I_{2}$,

$B_{1}=r_{2}-\sigma_{2}-2 v y_{2}-n_{2} x_{2}$.

$\lambda_{1}=\frac{\sigma \beta x_{2}}{\alpha^{\prime}+x_{2}}-\left(\delta I_{2}+\mu+q_{2} E_{2}\right)<0 \quad$ if $\quad x_{2}<\frac{\alpha^{\prime}\left(\delta I_{2}+\mu+q_{2} E_{2}\right)}{\sigma \beta-\alpha^{\prime}\left(\delta I_{2}+\mu+q_{2} E_{2}\right)}$,

then $\left|\arg \left(\lambda_{1}\right)\right|=\pi>\frac{\alpha \pi}{2}$.

We define the discriminant of polynomial $P(\lambda)=\lambda^{3}+c_{2} \lambda^{2}+c_{1} \lambda+c_{0}$ in this form [2]:

$D(P)=18 c_{1} c_{2} c_{0}+\left(c_{2} c_{1}\right)^{2}-4 c_{1}^{3}-4 c_{2}^{3} c_{0}-27 c_{0}^{2}$.

(1) If $D(P)>0, P_{2}$ is asymptotically stable if $c_{0}, c_{1}, c_{2}>0$ and $c_{2} c_{1}-c_{0}>0$ for all $\alpha \in[0,1[$.

If $D(P)<0$ and $c_{0}, c_{1}, c_{2}>0 \quad P_{2}$ is asymptotically stable if $\alpha<\frac{2}{3}$ and $c_{2} c_{1}-c_{0}>0$.

If $D(P)<0, c_{0}, c_{1}, c_{2}>0$ and $c_{2} c_{1}=c_{0}, P_{2}$ is asymptotically stable for all $\alpha \in[0,1[$.

Theorem 2.6. The equilibrium point $P_{3}\left(x_{3}, y_{3}, S_{3}, 0\right)$ is locally asymptotically stable if $\delta S_{3}+\sigma \gamma x_{3}<\eta+q_{3} E_{3}$ and (2.18) are satisfied.

Proof. The characteristic equation of matrix $J\left(x_{3}, y_{3}, S_{3}, 0\right)$ :

$\left(\lambda-\left(\delta S_{3}+\sigma \gamma x_{3}-\eta-q_{3} E_{3}\right)\right)\left(\lambda^{3}+d_{2} \lambda^{2}+d_{1} \lambda+d_{0}\right)=0$, where:

$d_{2}=-\left(A_{2}+B_{2}\right)$,

$d_{1}=A_{2} B_{2}-\left(\sigma_{1}-n_{2} y_{3}\right)\left(\sigma_{2}-n_{1} x_{3}\right)+\frac{\sigma \beta^{2} \alpha^{\prime} x_{3} S_{3}}{\left(\alpha^{\prime}+x_{3}\right)^{3}}$,

$d_{0}=-B_{2} \frac{\sigma \beta^{2} \alpha^{\prime} x_{3} S_{3}}{\left(\alpha^{\prime}+x_{3}\right)^{3}}$,

$A_{2}=r_{1}-\sigma_{1}-q_{1} E_{1}-2 \frac{r_{1}+K u}{K} x_{3}-n_{1} y_{3}-\frac{\beta \alpha^{\prime} S_{3}}{\left(\alpha^{\prime}+x_{3}\right)^{2}}$,

$B_{2}=r_{2}-\sigma_{2}-2 v y_{3}-n_{2} x_{3}$.

The first eigenvalues $\lambda_{1}=\delta S_{3}+\sigma \gamma x_{3}-\eta-q_{3} E_{3}<0$ if $\delta S_{3}+\sigma \gamma x_{3}<\eta+q_{3} E$, then $\left|\arg \left(\lambda_{1}\right)\right|=\pi>\frac{\alpha \pi}{2}$.

We define the discriminant of polynomial $Q(\lambda)=\lambda^{3}+d_{2} \lambda^{2}+d_{1} \lambda+d_{0}$ in this form [2]:

$D(Q)=18 d_{1} d_{2} d_{0}+\left(d_{2} d_{1}\right)^{2}-4 d_{1}^{3}-4 d_{2}^{3} d_{0}-27 d_{0}^{2}$.

(1) If $D(Q)>0, \quad P_{3}$ is asymptotically stable if $d_{0}, d_{1}, d_{2}>0$ and $d_{2} d_{1}-d_{0}>0$

for all $\alpha \in[0,1[$.

(2) If $D(Q)<0$ and $d_{0}, d_{1}, d_{2}>0 \quad P_{3}$ is asymptotically stable if $\alpha<\frac{2}{3}$ and $c_{2} c_{1}-c_{0}>0$.

(3) If $D(Q)<0, d_{0}, d_{1}, d_{2}>0$ and $d_{2} d_{1}=d_{0}, P_{3}$ is asymptotically stable

for all $\alpha \in[0,1[$. 
Theorem 2.7. The equilibrium point $P_{4}\left(x_{4}, y_{4}, S_{4}, I_{4}\right)$ is locally asymptotically stable if $\phi_{0}, \phi_{1}, \phi_{2}, \phi_{3}>0$, and $\phi_{2} \phi_{3}-\phi_{1}>\frac{\phi_{0} \phi_{3}^{2}}{\phi_{1}}$.

Proof. From the Jacobian $J\left(x_{4}, y_{4}, S_{4}, I_{4}\right)$ matrix, the characteristic equation of $P_{4}$ is:

$$
R(\lambda)=\lambda^{4}+\phi_{3} \lambda^{3}+\phi_{2} \lambda^{2}+\phi_{1} \lambda+\phi_{0}
$$

where:

$$
\begin{aligned}
\phi_{3} & =-\left(a_{6}+f_{6}\right), \\
\phi_{2} & =a_{6} f_{6}-b_{6} e_{6}-c_{6} g_{6}-d_{6} i_{6}-h_{6} j_{6}, \\
\phi_{1} & =a_{6} h_{6} j_{6}+c_{6} f_{6} g_{6}+d_{6} f_{6} i_{6}+f_{6} h_{6} j_{6}-i_{6} c_{6} h_{6}-d_{6} g_{6} j_{6}, \\
\phi_{0} & =j_{6} b_{6} e_{6} h_{6}+i_{6} c_{6} f_{6} h_{6}+d_{6} f_{6} g_{6} j_{6}-j_{6} a_{6} f_{6} h_{6}, \\
a_{6} & =r_{1}-\sigma_{1}-q_{1} E_{1}-2\left(\frac{r_{1}}{K}+u\right) x_{4}-n_{1} y_{4}-\gamma I_{4}-\frac{\beta \alpha^{\prime} S_{4}}{\left(\alpha^{\prime}+x_{4}\right)^{2}}-\gamma I_{4}, \\
b_{6} & =\sigma_{2}-n_{1} x_{4}, \\
c_{6} & =\frac{-\beta x_{4}}{\alpha^{\prime}+x_{4}}, \\
d_{6} & =-\gamma x_{4}, \\
e_{6} & =\sigma_{1}-n_{2} y_{4}, \\
f_{6} & =r_{2}-\sigma_{2}-2 v y_{4}-n_{2} x_{4}, \\
g_{6} & =\frac{\sigma \beta \alpha^{\prime} S_{4}}{\left(\alpha^{\prime}+x_{4}\right)^{2}}, \\
h_{6} & =-\delta S_{4}, \\
i_{6} & =\sigma \gamma I_{4}, \\
j_{6} & =\delta I_{4} .
\end{aligned}
$$

We pose the discriminant $D(R)$ in following form:

$$
\begin{aligned}
D(R)= & 256 \phi_{0}^{3}-192 \phi_{3} \phi_{1} \phi_{0}^{2}-128 \phi_{2}^{2} \phi_{0}+144 \phi_{2} \phi_{1}^{2} \phi_{0}-27 \phi_{1}^{4}+144 \phi_{3}^{2} \phi_{2} \phi_{0}^{2} \\
& -6 \phi_{3}^{2} \phi_{1}^{2} \phi_{0}-80 \phi_{3} \phi_{2}^{2} \phi_{1} \phi_{0}+18 \phi_{3} \phi_{2} \phi_{1}^{3}+16 \phi_{2}^{4} \phi_{0}-4 \phi_{2}^{3} \phi_{1}^{2}-27 \phi_{3}^{4} \phi_{0}^{2} \\
& +18 \phi_{3}^{3} \phi_{2} \phi_{1} \phi_{0}-4 \phi_{3}^{3} \phi_{1}^{3}-4 \phi_{3}^{2} \phi_{2}^{3} \phi_{0}+\left(\phi_{3} \phi_{2} \phi_{1}\right)^{2} .
\end{aligned}
$$

Using the results of [2],

(1) If $D(R)>0, \phi_{0}, \phi_{1}, \phi_{2}, \phi_{3}>0$ and $\phi_{2} \phi_{3}-\phi_{1}>\frac{\phi_{0} \phi_{3}^{2}}{\phi_{1}}, P_{4}$ is locally asymptotically stable for all $\alpha \in[0,1[$.

(2) If $D(R)<0, \phi_{0}, \phi_{1}, \phi_{2}, \phi_{3}>0$ and $\alpha<\frac{1}{3}, P_{4}$ is locally asymptotically stable.

(3) If $D(R)<0, \phi_{0}, \phi_{1}, \phi_{2}, \phi_{3}>0$ and $\phi_{2}=\frac{\phi_{3} \phi_{0}}{\phi_{1}}+\frac{\phi_{1}}{\phi_{3}}, \quad P_{4}$ is locally asymptotically stable for all $\alpha \in[0,1[$.

\section{Global stability of EQUilibira}

In this section, we prove the global stability of each equilibrium point of system (1.1) using Lyapunov functions. 
Theorem 3.1. The equilibrium $P_{1}\left(x_{1}, y_{1}, 0,0\right)$ is globally asymptotically stable if (2.5), (2.6), (2.7) and $n_{1}+$ $\frac{\sigma_{2} n_{2} y_{1}}{\sigma_{1} x_{1}}<2 \min \left(\frac{\sigma_{2} y_{1} v}{\sigma_{1} x_{1}}, \frac{r_{1}}{K}+u\right)$ are realized.

Proof. Consider the following positive definite Lyapunov function about $P_{1}\left(x_{1}, y_{1}, 0,0\right)$ : $V_{1}(x, y)=\left(x-x_{1}-x_{1} \ln \left(\frac{x}{x_{1}}\right)\right)+\frac{\sigma_{2} y_{1}}{\sigma_{1} x_{1}}\left(y-y_{1}-y_{1} \ln \left(\frac{y}{y_{1}}\right)\right)$.

Using [28], we get:

$$
\begin{aligned}
D^{\alpha} V_{1} \leq & \frac{x-x_{1}}{x} D^{\alpha} x+\frac{\sigma_{2} y_{1}}{\sigma_{1} x_{1}}\left(\frac{y-y_{1}}{y}\right) D^{\alpha} y \\
\leq & \left(x-x_{1}\right)\left(-\left(\frac{r_{1}+K u}{K}\right)\left(x-x_{1}\right)+\sigma_{2}\left(\frac{y}{x}-\frac{y_{1}}{x_{1}}-n_{1}\left(y-y_{1}\right)\right)\right) \\
& +\frac{\sigma_{2} y_{1}}{\sigma_{1} x_{1}}\left(y-y_{1}\right)\left(-v\left(y-y_{1}\right)+\sigma_{1}\left(\frac{x}{y}-\frac{x_{1}}{y_{1}}\right)-n_{2}\left(x-x_{1}\right)\right) .
\end{aligned}
$$

Using $-\left(x-x_{1}\right)\left(y-y_{1}\right)<\frac{1}{2}\left(\left(x-x_{1}\right)^{2}+\left(y-y_{1}\right)^{2}\right)$ we find,

$$
\begin{aligned}
D^{\alpha} V_{1} \leq & -\left(\frac{r_{1}+K u}{K}\right)\left(x-x_{1}\right)^{2}-\frac{v \sigma_{2} y_{1}}{\sigma_{1} x_{1}}\left(y-y_{1}\right)^{2}-\frac{\sigma_{2}}{x x_{1} y}\left(y x_{1}-y_{1} x\right)^{2} \\
& -\left(n_{1}+\frac{\sigma_{2} y_{1} n_{2}}{\sigma_{1} x_{1}}\right)\left(x-x_{1}\right)\left(y-y_{1}\right), \\
\leq & \left(\frac{1}{2}\left(n_{1}+\frac{\sigma_{2} y_{1} n_{2}}{\sigma_{1} x_{1}}\right)-\frac{r_{1}+K u}{K}\right)\left(x-x_{1}\right)^{2}+\left(\frac{1}{2}\left(n_{1}+\frac{\sigma_{2} y_{1} n_{2}}{\sigma_{1} x_{1}}\right)-\frac{v \sigma_{2} y_{1}}{\sigma_{1} x_{1}}\right)\left(y-y_{1}\right)^{2} \\
& -\frac{\sigma_{2}}{x x_{1} y}\left(y x_{1}-y_{1} x\right)^{2} .
\end{aligned}
$$

Therefore, $D^{\alpha} V_{1}<0$ if $n_{1}+\frac{\sigma_{2} n_{2} y_{1}}{\sigma_{1} x_{1}}<2 \min \left(\frac{\sigma_{2} y_{1} v}{\sigma_{1} x_{1}}, \frac{r_{1}}{K}+u\right)$.

Theorem 3.2. The equilibrium $P_{2}\left(x_{2}, y_{2}, 0, I_{2}\right)$ is globally asymptotically stable if (2.9) and $n_{1}+\frac{\sigma_{2} n_{2} y_{2}}{\sigma_{1} x_{2}}<$ $2 \min \left(\frac{\sigma_{2} y_{2} v}{\sigma_{1} x_{2}}, \frac{r_{1}}{K}+u\right)$ are realized.

Proof. Consider the following positive definite Lyapunov function about $P_{2}\left(x_{2}, y_{2}, 0, I_{2}\right)$ :

$V_{2}(x, y, I)=\left(x-x_{2}-x_{2} \ln \left(\frac{x}{x_{2}}\right)\right)+\frac{\sigma_{2} y_{2}}{\sigma_{1} x_{2}}\left(y-y_{2}-y_{2} \ln \left(\frac{y}{y_{2}}\right)\right)+\frac{1}{\sigma}\left(I-I_{2}-I_{2} \ln \left(\frac{I}{I_{2}}\right)\right)$.

Using [28] we get:

$$
\begin{aligned}
D^{\alpha} V_{2} \leq & \left(x-x_{2}\right)\left(-\left(\frac{r_{1}+K u}{K}\right)\left(x-x_{2}\right)+\sigma_{2}\left(\frac{y}{x}-\frac{y_{2}}{x_{2}}\right)-n_{1}\left(y-y_{2}\right)-\gamma\left(I-I_{2}\right)\right) \\
& +\frac{\sigma_{2} y_{2}}{\sigma_{1} x_{2}}\left(y-y_{2}\right)\left(-v\left(y-y_{2}\right)+\sigma_{1}\left(\frac{x}{y}-\frac{x_{2}}{y_{2}}\right)-n_{2}\left(x-x_{2}\right)\right)+\left(I-I_{2}\right) \gamma\left(x-x_{2}\right), \\
\leq & -\frac{v \sigma_{2} y_{2}}{\sigma_{2} y_{2}}\left(y-y_{2}\right)^{2}-\frac{\sigma_{2}}{x x_{2} y}\left(y x_{2}-y_{2} x\right)^{2}-\left(n_{1}+\frac{\sigma_{2} y_{2} n_{2}}{\sigma_{1} x_{2}}\right)\left(x-x_{2}\right)\left(y-y_{2}\right), \\
\leq & \left(\frac{1}{2}\left(n_{1}+\frac{\sigma_{2} y_{2} n_{2}}{\sigma_{1} x_{2}}\right)-\frac{r_{1}+K u}{K}\right)\left(x-x_{2}\right)^{2}-\left(\frac{1}{2}\left(n_{1}+\frac{n_{2} \sigma_{2} y_{2}}{\sigma_{1} x_{2}}\right)-\frac{v \sigma_{2} y_{2}}{\sigma_{1} x_{2}}\right)\left(y-y_{2}\right)^{2} \\
& -\frac{\sigma_{2}}{x x_{2} y}\left(y x_{2}-y_{2} x\right)^{2} .
\end{aligned}
$$

Therefore, $D^{\alpha} V_{2}<0$ if $n_{1}+\frac{\sigma_{2} n_{2} y_{2}}{\sigma_{1} x_{2}}<2 \min \left(\frac{\sigma_{2} y_{2} v}{\sigma_{1} x_{2}}, \frac{r_{1}}{K}+u\right)$.

Theorem 3.3. The equilibrium $P_{3}\left(x_{3}, y_{3}, S_{3}, 0\right)$ is globally asymptotically stable if (2.10) and $n_{1}+\frac{\sigma_{2} n_{2} y_{3}}{\sigma_{1} x_{3}}<$ $2 \min \left(\frac{\sigma_{2} y_{3} v}{\sigma_{1} x_{3}}, \frac{r_{1}}{K}+u-\frac{\beta S_{3}}{\alpha^{\prime}\left(\alpha^{\prime}+x_{3}\right)}\right)$ are realized.

Proof. Consider the following positive definite Lyapunov function about $P_{3}\left(x_{3}, y_{3}, S_{3}, 0\right)$ :

$$
\begin{aligned}
V_{3}(x, y, S)= & \left(x-x_{3}-x_{3} \ln \left(\frac{x}{x_{3}}\right)\right)+\frac{\sigma_{2} y_{3}}{\sigma_{1} x_{3}}\left(y-y_{3}-y_{3} \ln \left(\frac{y}{y_{3}}\right)\right) \\
& +\frac{\alpha^{\prime}+x_{3}}{\alpha^{\prime} \sigma}\left(S-S_{3}-S_{3} \ln \left(\frac{S}{S_{3}}\right)\right) .
\end{aligned}
$$

Using [28], we obtain: 


$$
\begin{aligned}
D^{\alpha} V_{3} \leq & \left(x-x_{3}\right)\left(-\left(\frac{r_{1}+K u}{K}\right)\left(x-x_{3}\right)+\sigma_{2}\left(\frac{y}{x}-\frac{y_{3}}{x_{3}}\right)-n_{1}\left(y-y_{3}\right)-\beta\left(\frac{S}{\alpha^{\prime}+x}-\frac{S_{3}}{\alpha^{\prime}+x_{3}}\right)\right) \\
& +\frac{\sigma_{2} y_{3}}{\sigma_{1} x_{3}}\left(y-y_{1}\right)\left(-v\left(y-y_{1}\right)+\sigma_{1}\left(\frac{x}{y}-\frac{x_{1}}{y_{1}}\right)-n_{2}\left(x-x_{2}\right)\right) \\
& +\frac{\alpha^{\prime}+x_{3}}{\alpha^{\prime} \sigma}\left(S-S_{3}\right) \sigma \beta\left(\frac{x}{x+\alpha^{\prime}}-\frac{x_{3}}{\alpha^{\prime}+x_{3}}\right), \\
\leq \quad & -\left(\frac{r_{1}+K u}{K}\right)\left(x-x_{3}\right)^{2}-\frac{v \sigma_{2} y_{3}}{\sigma_{1} x_{3}}\left(y-y_{3}\right)^{2}-\frac{\sigma_{2}}{x x_{3} y}\left(y x_{3}-y_{3} x\right)^{2} \\
& -\left(n_{1}+\frac{\sigma_{2} y_{3} n_{2}}{\sigma_{1} x_{3}}\right)\left(x-x_{3}\right)\left(y-y_{3}\right)+\frac{\beta S_{3}\left(x-x_{3}\right)^{2}}{\left(\alpha^{\prime}+x\right)\left(\alpha^{\prime}+x_{3}\right)} \\
\leq \quad & \left(\frac{1}{2}\left(n_{1}+\frac{\sigma_{2} y_{3} n_{2}}{\sigma_{1} x_{3}}\right)-\frac{r_{1}+K u}{K}+\frac{\beta S_{3}}{\alpha^{\prime}\left(\alpha^{\prime}+x_{3}\right)}\right)\left(x-x_{3}\right)^{2} \\
& -\left(\frac{1}{2}\left(n_{1}+\frac{\sigma_{2} y_{3} n_{2}}{\sigma_{1} x_{3}}\right)-\frac{v \sigma_{2} y_{3}}{\sigma_{1} x_{3}}\right)\left(y-y_{3}\right)^{2}-\frac{\sigma_{2}}{x x_{3} y}\left(y x_{3}-y_{3} x\right)^{2} .
\end{aligned}
$$

Therefore, $D^{\alpha} V_{3}<0$ if $n_{1}+\frac{\sigma_{2} n_{2} y_{3}}{\sigma_{1} x_{3}}<2 \min \left(\frac{\sigma_{2} y_{3} v}{\sigma_{1} x_{3}}, \frac{r_{1}}{K}+u-\frac{\beta S_{3}}{\alpha^{\prime}\left(\alpha^{\prime}+x_{3}\right)}\right)$.

Theorem 3.4. The equilibrium $P_{4}\left(x_{4}, y_{4}, S_{3}, I_{4}\right)$ is globally asymptotically stable if (2.11) and $n_{1}+\frac{\sigma_{2} n_{2} y_{4}}{\sigma_{1} x_{4}}<$ $2 \min \left(\frac{\sigma_{2} y_{4} v}{\sigma_{1} x_{4}}, \frac{r_{1}}{K}+u+\frac{\beta\left(x_{4}-2 S_{4}\right)}{2\left(\alpha^{\prime}+x_{4}\right)}\right)$ are realized.

Proof. Consider the following positive definite Lyapunov function about $P_{4}\left(x_{4}, y_{4}, S_{4}, I_{4}\right)$ :

$$
\begin{aligned}
V_{4}(x, y, S, I)= & \left(x-x_{4}-x_{4} \ln \left(\frac{x}{x_{4}}\right)\right)+\frac{\sigma_{2} y_{4}}{\sigma_{1} x_{4}}\left(y-y_{4}-y_{4} \ln \left(\frac{y}{y_{4}}\right)\right) \\
& +\frac{1}{\sigma}\left(S-S_{4}-S_{4} \ln \left(\frac{S}{S_{4}}\right)\right)+\frac{1}{\sigma}\left(I-I_{4}-I_{4} \ln \left(\frac{I}{I_{4}}\right)\right) .
\end{aligned}
$$

Using [28], we obtain:

$$
\begin{aligned}
D^{\alpha} V_{4} \leq \quad & \left(x-x_{4}\right)\left(-\left(\frac{r_{1}+K u}{K}\right)\left(x-x_{4}\right)+\sigma_{2}\left(\frac{y}{x}-\frac{y_{4}}{x_{4}}\right)-n_{1}\left(y-y_{4}\right)-\beta\left(\frac{S}{\alpha^{\prime}+x}-\frac{S_{4}}{\alpha^{\prime}+x_{4}}\right)-\gamma\left(I-I_{4}\right)\right) \\
& +\frac{\sigma_{2} y_{4}}{\sigma_{1} x_{4}}\left(y-y_{4}\right)\left(-v\left(y-y_{4}\right)+\sigma_{1}\left(\frac{x}{y}-\frac{x_{4}}{y_{4}}\right)-n_{2}\left(x-x_{4}\right)\right) \\
& +\frac{1}{\sigma}\left(S-S_{4}\right)\left(\sigma \beta\left(\frac{x}{x+\alpha^{\prime}}-\frac{x_{4}}{\alpha^{\prime}+x_{4}}\right)-\delta\left(I-I_{4}\right)\right)+\frac{\left(I-I_{4}\right)\left(\sigma \gamma\left(x-x_{4}\right)+\delta\left(S-S_{4}\right)\right)}{\sigma}, \\
\leq \quad & -\left(\frac{r_{1}+K u}{K}\right)\left(x-x_{4}\right)^{2}-\frac{v \sigma_{2} y_{4}}{\sigma_{1} x_{4}}\left(y-y_{4}\right)^{2}-\frac{\sigma_{2}}{x x_{4} y}\left(y x_{4}-y_{4} x\right)^{2} \\
& -\left(n_{1}+\frac{\sigma_{2} y_{4} n_{2}}{\sigma_{1} x_{4}}\right)\left(x-x_{4}\right)\left(y-y_{4}\right)-\frac{\beta\left(x-x_{4}\right)}{\left(\alpha^{\prime}+x\right)\left(\alpha^{\prime}+x_{4}\right)}\left(S x_{4}-x S_{4}\right), \\
\leq \quad & \left(\frac{1}{2}\left(n_{1}+\frac{\sigma_{2} y_{4} n_{2}}{\sigma_{1} x_{4}}\right)-\frac{r_{1}+K u}{K}+\frac{\beta S_{4}}{\alpha^{\prime}\left(\alpha^{\prime}+x_{4}\right)}-\frac{\beta x_{4}}{2\left(\alpha^{\prime}+x_{4}\right)}\right)\left(x-x_{4}\right)^{2}-\frac{\sigma_{2}}{x x_{4} y}\left(y x_{4}-y_{4} x\right)^{2} \\
& +\left(\frac{1}{2}\left(n_{1}+\frac{\sigma_{2} y_{4} n_{2}}{\sigma_{1} x_{4}}\right)-\frac{v \sigma_{2} y_{4}}{\sigma_{1} x_{4}}\right)\left(y-y_{4}\right)^{2}-\frac{\beta x_{4}}{\left(\alpha^{\prime}+x\right)\left(\alpha^{\prime}+x_{4}\right)}\left(S-S_{4}\right)^{2} .
\end{aligned}
$$

Therefore, $D^{\alpha} V_{4}<0$ if $n_{1}+\frac{\sigma_{2} n_{2} y_{4}}{\sigma_{1} x_{4}}<2 \min \left(\frac{\sigma_{2} y_{4} v}{\sigma_{1} x_{4}} ; \frac{r_{1}}{K}+u+\frac{\beta\left(x_{4}-2 S_{4}\right)}{2\left(\alpha^{\prime}+x_{4}\right)}\right)$.

\section{Numerical SIMULATIONS}

To show the influence of the parameter $\alpha$ on our fractional order model, we take the different values of $\alpha$ in numerical simulations of the curves $x(t), y(t), S(t)$ and $I(t)$ that are shown in Figures 2-5. These figures show that the system (1.1) reaches the equilibrium state for the different values of $\alpha$. These results show the effectiveness of Theorems 2.4-2.7. As we can see, numerical solutions are permanently dependent on the fractional order derivative $\alpha$ and the model reaches the equilibrium point more rapidly by reducing $\alpha$. In other words, the model approaches the steady state more quickly when the memory factor effect is increased.

To demonstrate the theoretical results obtained in this paper, we give some numerical simulations. We consider the parameters values as given by [14]:

$r_{1}=5, r_{2}=1, n_{1}=0.5, n_{2}=0.3, \sigma_{1}=1, \sigma_{2}=0.9, q_{1}=0.1, q_{2}=0.2, q_{3}=0.4, E_{1}=5, E_{2}=5.2 ; E_{3}=4.8$, $u=0.0001, v=0.333, K=4, \alpha^{\prime}=0.7, \beta=0.94, \sigma=0.998, \delta=10, \mu=1, \gamma=5.5, \eta=60$, with initial conditions $(x(0), y(0), S(0), I(0))=(1,1,1,1)$, so $P_{1}$ is locally asymptotically stable.

As it's shown in this example the parameter values are chosen as:

$r_{1}=6, r_{2}=8, n_{1}=0.5, n_{2}=0.3, \sigma_{1}=2, \sigma_{2}=2, q_{1}=0.1, q_{2}=0.2, u=0.4, v=0.4, K=5, \alpha^{\prime}=0.45, \beta=1$, 

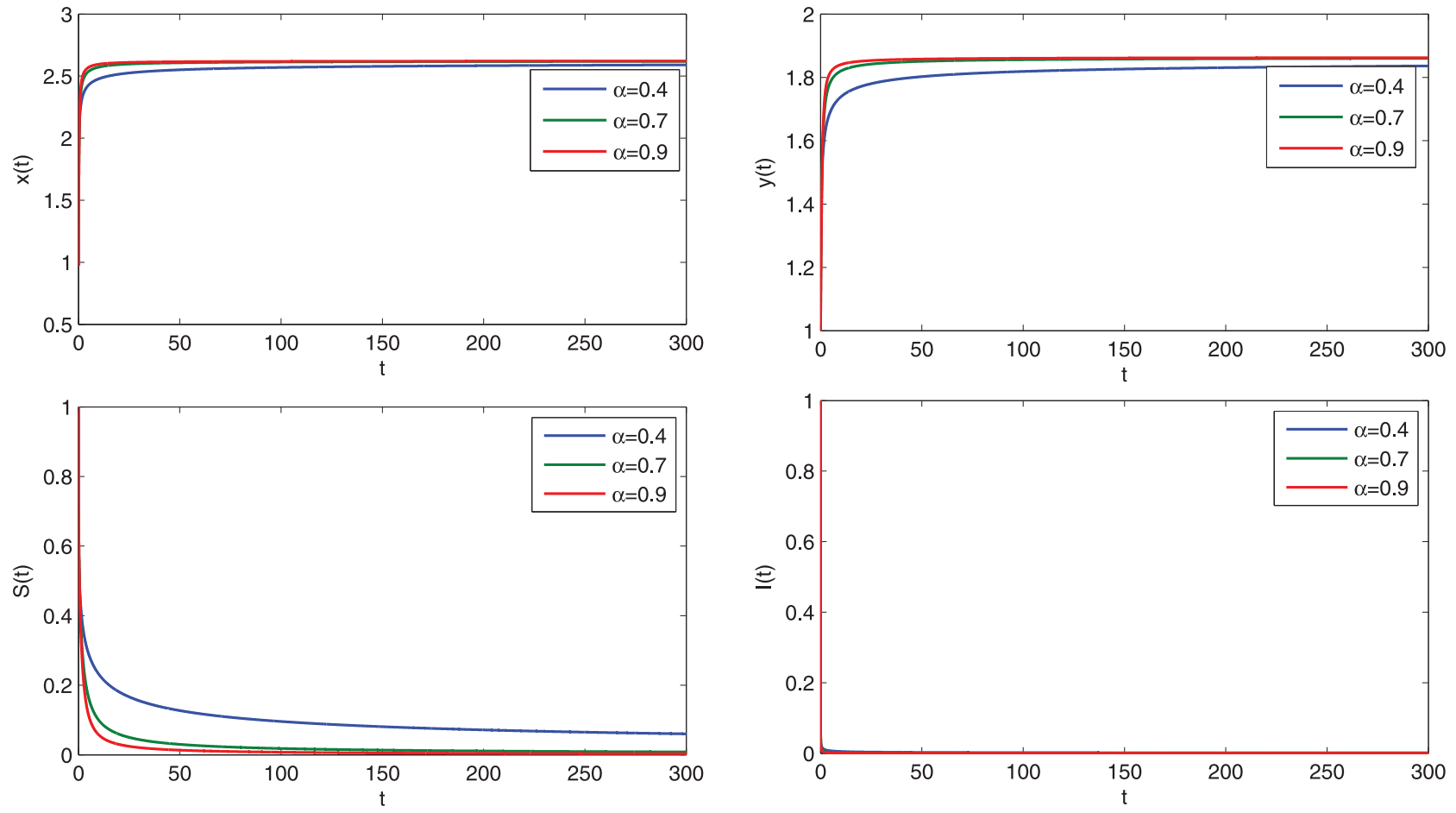

FiguRE 2. Solution curves corresponding to the set values parameters of the system (1.1) of equilibrium $P_{1}$ with different values of $\alpha$.
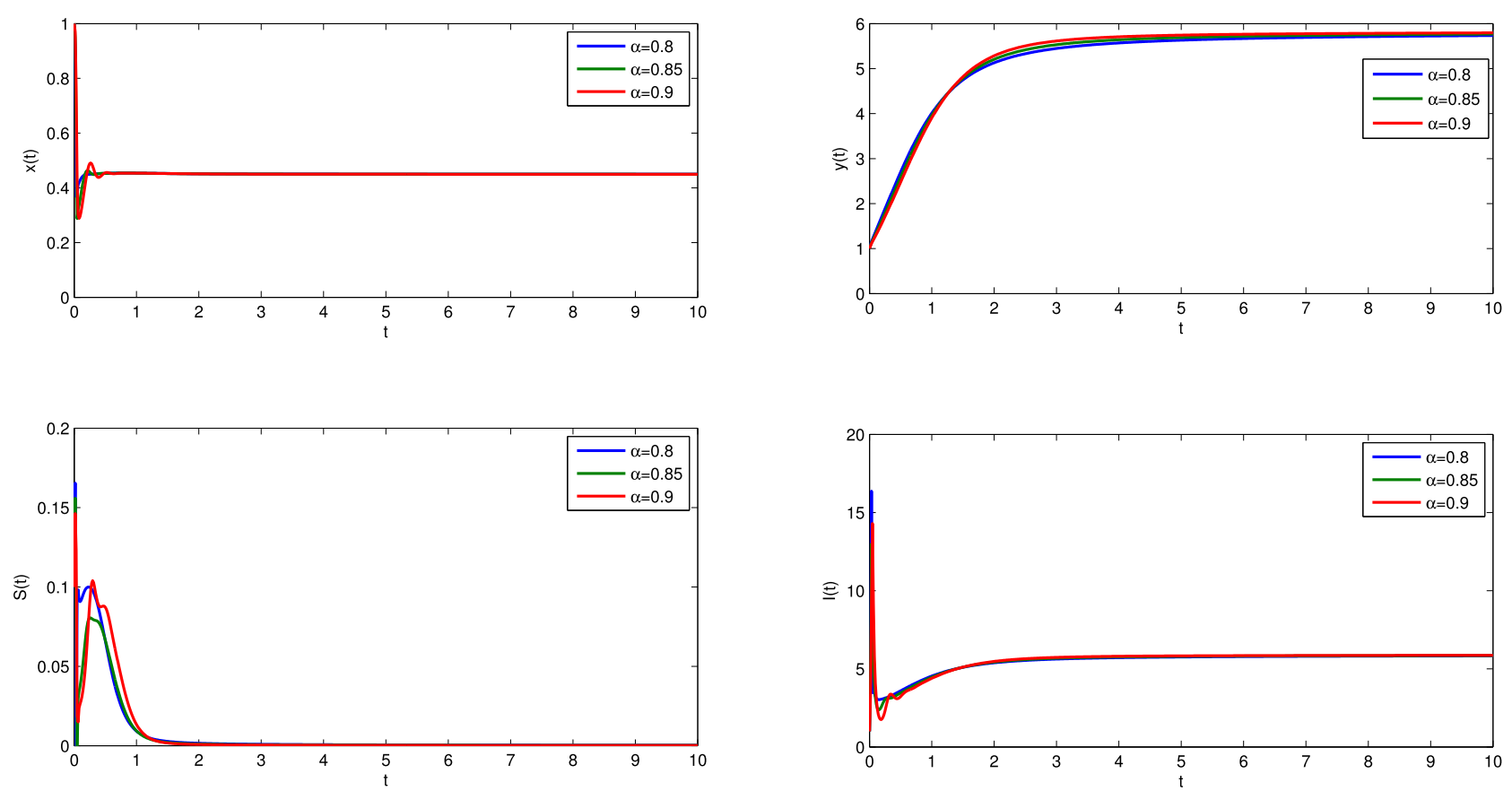

FiguRE 3. Solution curves corresponding to the set values parameters of the system (1.1) of equilibrium $P_{2}$ with different values of $\alpha$. 

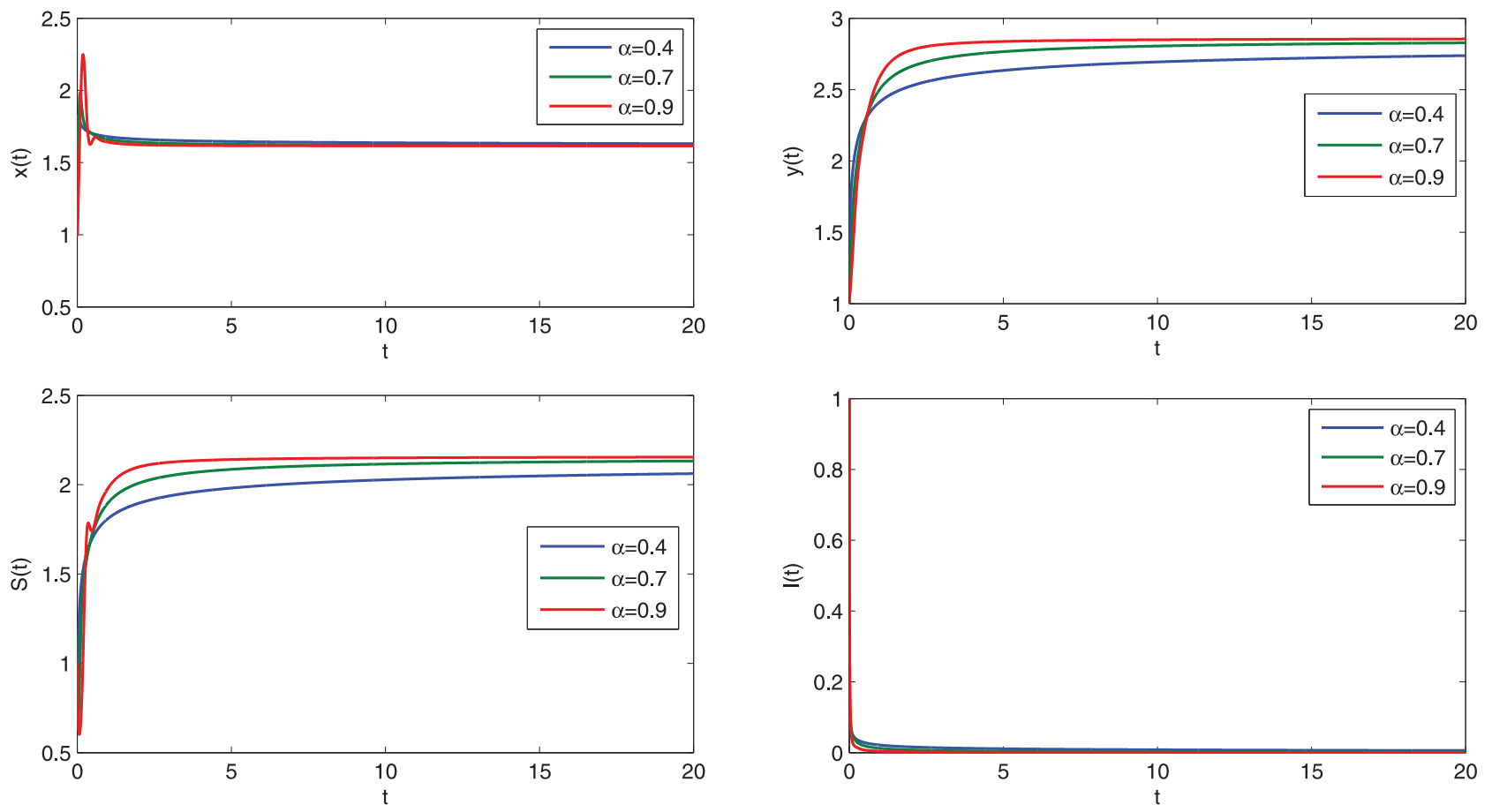

FiguRE 4. Solution curves corresponding to the set values parameters of the system (1.1) of equilibrium $P_{3}$ with different values of $\alpha$.
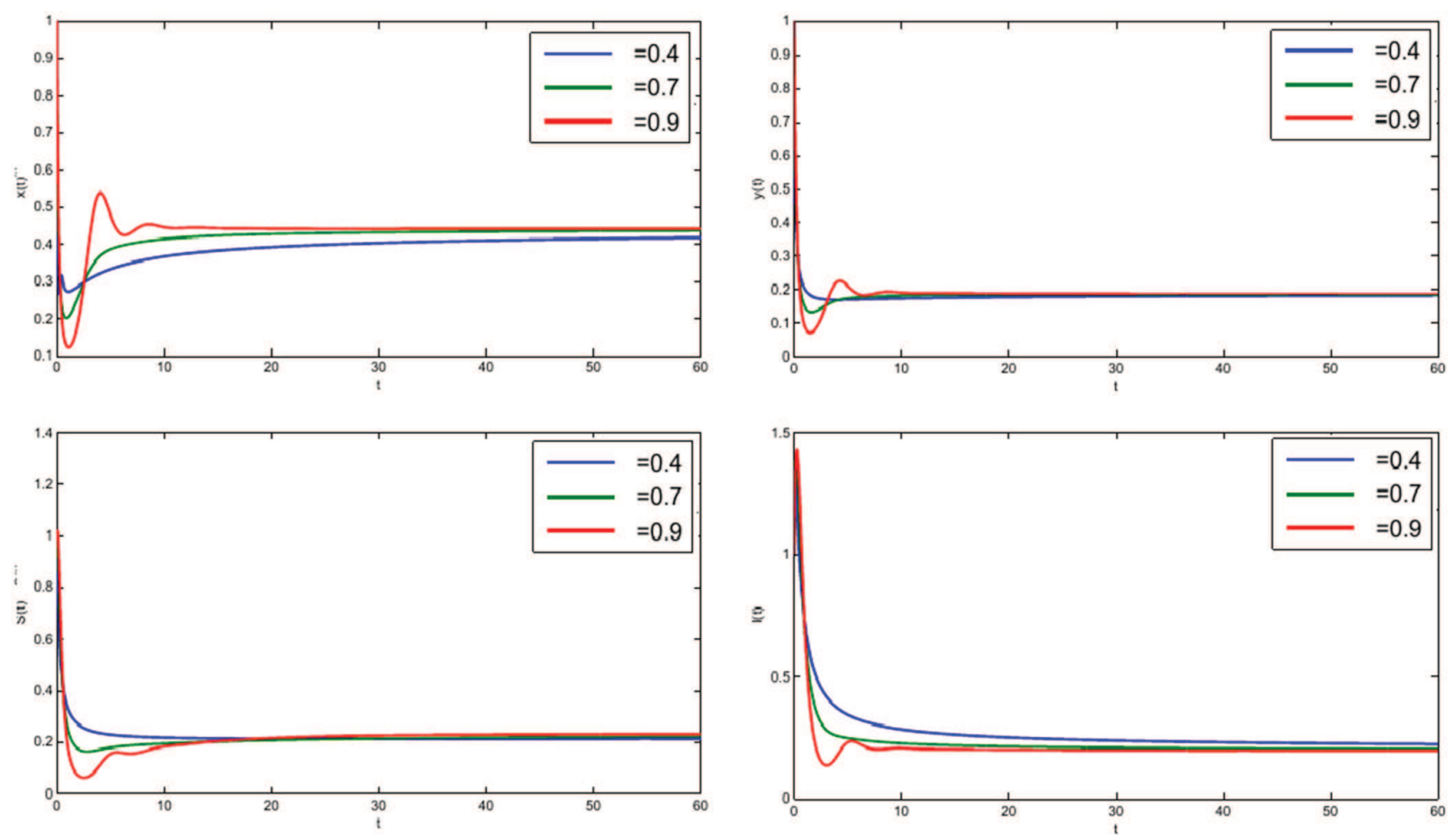

FiguRE 5. Solution curves corresponding to the set values parameters of the system (1.1) of equilibrium $P_{4}$ with different values of $\alpha$. 
$\sigma=4, \delta=10, \mu=1, \gamma=5.5, \eta=60$. The system (1.1) with initial conditions $(x(0), y(0), S(0), I(0))=$ $(1,1,0.1,1)$, so $P_{2}$ is locally asymptotically stable.

In this example, the parameter values are:

$r_{1}=10, r_{2}=8, n_{1}=0.5, n_{2}=0.3, \sigma_{1}=2, \sigma_{2}=7.5, q_{1}=0.1, q_{2}=0.2, q_{3}=0.5, E_{1}=3, E_{2}=3.2$,

$E_{3}=4, u=0.01, v=0.4, K=5, \alpha^{\prime}=5, \beta=50, \sigma=1.5, \delta=10, \mu=18, \gamma=5.33, \eta=60$, with initial conditions $(x(0), y(0), S(0), I(0))=(1,1,1,1)$, so $P_{3}$ is locally asymptotically stable.

In this example, the parameter values are:

$r_{1}=2.34, r_{2}=3, n_{1}=0.5, n_{2}=0.3, \sigma_{1}=2, \sigma_{2}=7.5, q_{1}=0.1, q_{2}=0.2, q_{3}=0.3, E_{1}=3, E_{2}=2.6, E_{3}=2.2$,

$u=0.01, v=0.4, K=0.7, \alpha^{\prime}=0.7, \beta=0.94, \sigma=0.998, \delta=1.34, \mu=2.41, \gamma=8, \eta=2.68$, with initial conditions $(x(0), y(0), S(0), I(0))=(1,1,1,1)$, so $P_{4}$ is locally asymptotically stable.

We observe from simulations, the effect of reducing the order of the time derivative can be observed. As the fractional order $\alpha$ decreases, the system (with Caputo derivative) stabilizes more quickly. It is the largest "memory" of the system of past states, the greater the damping of the oscillations in the dynamics of the system. The simulations show that, even with fairly moderate reductions in $\alpha$, the amplitude of the population density oscillations is greatly delayed.

\section{CONCLUSiOn}

In this paper, we investigated a Dynamics of the fractional order prey-predator model in the presence of competition and toxicity using the Caputo fractional derivative. We have established the existence and boundedness of the solutions. After calculating the equilibrium of our model under certain conditions, we have analyzed the local stability using Matiginon's conditions [17]. Global stability has been studied using Lyapunov functions. From our numerical results, we can observe that the different values of $\alpha$ have no effect on the stability of equilibria but have an effect on the time necessary to achieve equilibrium states. These variations are verified in the numerical simulations illustrated in the Figures 2-5, as the curves $x, y, S$ and $I$ that converge towards the equilibrium points. Finally, we can conclude that the memory effect of the fractional order derivative affects the dynamics of our proposed system.

\section{REFERENCES}

[1] E. Ahmed and A. Elgazzar, On fractional order differential equations model for nonlocal epidemics. Physica A 379 (2007) 607-614.

[2] E. Ahmed, E.-S. Ama, El-Saka and A.A. Hala, On some Routh-Hurwitz conditions for fractional order differential equations and their applications in Lorenz, Rässler, Chua and Chen systems. Phys. Lett. A 358 (2006) 1-4.

[3] A.A. Berryman, The origins and evolutions of predator-prey theory. Ecology 73 (1992) 1530-1535.

[4] K. Chakraborty and K. Das, Modeling and analysis of a two-zooplankton one-phytoplankton system in the presence of toxicity. Appl. Math. Model. 39 (2015) 1241-1265.

[5] D.R. Curtiss, Recent extentions of descartes rule of signs. Ann. Math. (1918) 251-278.

[6] T. Das, R.N. Mukherjee and K.S. Chaudhuri, Harvesting of a prey-predator fishery in the presence of toxicity. Appl. Math. Model. 33 (2009) 2282-2292.

[7] B. Dubey, A prey-predator model with a reserved area. Nonlinear Anal. Model. Control. 12 (2007) 479-494.

[8] B. Dubey, P. Chandra and P. Sinha, A model for fishery resource with reserve area. Nonlinear Anal. Real World Appl. 4 (2003) 625-637.

[9] M. Edelman, Fractional maps as maps with power-law memory. Nonlinear dynamics and complexity. Springer, Cham (2014) $79-120$.

[10] A. Elsadany and A. Matouk, Dynamical behaviors of fractional-order Lotka-Volterra predator-prey model and its discretization. J. Appl. Math. Comput. 49 (2015) 269-283.

[11] S. Jana, A. Ghorai, S. Guria and T.K. Kar, Global dynamics of a predator weaker prey and stronger prey system. Appl. Math. Comput. 250 (2015) 235-248.

[12] T.K. Kar, A model for fishery resource with reserve area and facing prey predator interactions. Can. Appl. Math. Quart. 14 (2006) 385-399.

[13] Y. Li, Y. Chen and I. Podlubny, Stability of fractional-order nonlinear dynamic systems: Lyapunov direct method and generalized Mittag-Leffler stability. Comp. Math. Appl. 59 (2010) 1810-1821.

[14] Y. Louartassi, A. Alla, K. Hattaf and A. Nabil, Dynamics of a predator-prey model with harvesting and reserve area for prey in the presence of competition and toxicity. J. Appl. Math. Comput. 1 (2018) 305-321. 
[15] Y. Louartassi, E. El Mazoudi and N. Elalami, A new generalization of lemma Gronwall-Bellman. Appl. Math. Sci. 6 (2012) 621-628.

[16] R.L. Magin, Fractional calculus in bioengineering. CRC Crit. Rev. Biomed. Eng. 32 (2004) 1-377.

[17] D. Matignon, Stability results for fractional differential equations with applications to control processing. Proc. Comput. Eng. Syst. Appl. Multiconf. 2 (1996) 963-968.

[18] R.M. May, Stability and complexity in model ecosystems. Princeton University Press, Princeton, New Jersey (1973).

[19] T.M. Michelitsch, G.A. Maugin, F.C.G.A. Nicolleau, A.F. Nowakowski and S. Derogar, Dispersion relations and wave operators in self-similar quasicontinuous linear chains. Phys. Rev. E 80 (2009) 011135.

[20] A. Mouaouine, A. Boukhouima, K. Hattaf and N. Yousfi, A fractional order SIR epidemic model with nonlinear incidence rate. Adv. Differ. Equ. 2018 (2018) 160.

[21] K. Oldham and J. Spanier, Vol. 111 of The fractional calculus theory and applications of differentiation and integration to arbitrary order. Elseiver, Amsterdam (1974).

[22] I. Podlubny, Vol. 198 of Differential Equations: An Introduction to Fractional Derivatives, Fractional Differential Equations, to methods of their Solution and Some of their applications. Elsevier, Amsterdam (1998).

[23] M. Riesz, L'intégrale de Riemann-Liouville et le probléme de Cauchy. Acta Math. 81 (1949) 1-222.

[24] B. Ross, S.G. Samko and E. Russel Love, Functions that have no First Order Derivative might have fractional derivatives of all orders less than one. Real Anal. Exchange 20 (1994) 140-157.

[25] M. Sambath, P. Ramesh and K. Balachandran, Asymptotic behavior of the fractional order three species prey-predator model. Int. J. Nonlinear Sci. Numer. Simul. 19 (2018) 721-733.

[26] S. Samko, A. Kilbas and O. Marichev, Fractional Integrals and Derivatives: Theory and Applications (1993).

[27] J.B. Shukla, A.K. Agrawal, B. Dubey and P. Sinha, Existence and survival of two competing species in a polluted environment: a mathematical model. J. Biol. Syst. 9 (2001) 89-103.

[28] C. Vargas De-León, Volterra-type Lyapunov functions for fractional-order epidemic systems. Commun. Nonlinear Sci. Numer. Simul. 24 (2015) 75-85.

[29] H. Yang and J. Jia, Harvesting of a predator-prey model with reserve area for prey and in the presence of toxicity. J. Appl. Math. Comput. 53 (2017) 693-708.

[30] X.Q. Zhao. Dynamical Systems in Population Biology. Springer New York (2000). 